\title{
EchoGéo
}

$8 \mid 2009$

Moyen-Orient : conflits et mobilités dans un espace mondialisé

\section{Commerce des produits d'élevage et territorialisation : une mise en débat à partir de trois travaux de géographes en Afrique}

Introduction et coordination du dossier

\section{Denis Gautier}

\section{(2) OpenEdition}

\section{Journals}

\section{Édition électronique}

URL : https://journals.openedition.org/echogeo/11090

DOI : 10.4000/echogeo. 11090

ISSN : 1963-1197

Éditeur

Pôle de recherche pour l'organisation et la diffusion de l'information géographique (CNRS UMR 8586)

\section{Référence électronique}

Denis Gautier, «Commerce des produits d'élevage et territorialisation : une mise en débat à partir de trois travaux de géographes en Afrique », EchoGéo [En ligne], 8 | 2009, mis en ligne le 26 mars 2009, consulté le 01 août 2021. URL : http://journals.openedition.org/echogeo/11090 ; DOI : https://doi.org/ 10.4000/echogeo. 11090

Ce document a été généré automatiquement le 1 août 2021.

EchoGéo est mis à disposition selon les termes de la licence Creative Commons Attribution - Pas d'Utilisation Commerciale - Pas de Modification 4.0 International (CC BY-NC-ND) 


\section{Commerce des produits d'élevage et territorialisation : une mise en débat à partir de trois travaux de géographes en Afrique}

Introduction et coordination du dossier

Denis Gautier

1 Le projet de recherche Icare, financé par le Cirad, a pour ambition d'analyser les liens entre la dynamique des marchés mondiaux et régionaux des produits animaux et le développement dans les territoires d'élevage. Les deux terrains d'application de ce projet sont l'Afrique de l'Ouest, en particulier le Sénégal et le Mali, et la Corne de l'Afrique.

2 Pendant longtemps, l'élevage de ces régions a eu la réputation d'être un élevage de prestige, voire un élevage contemplatif, pratiqué par des peuples d'éleveurs peu en prise avec les marchés (Faye, 2006). Les géographes qui ont étudiés ces régions (parmi lesquels on peut citer J. Gallais, D. Retaillé, M. D. Turner, T. Bassett, etc., pour l'Afrique de l'Ouest, A. Gascon ou J. Boutrais pour l'Afrique de l'Est) ainsi que les spécialistes des sciences sociales (B. Thébaut, A. Bourgeot, A. Marty, F. Vatin...) qui ont étudié ces peuples, nous donnent une image plus nuancée de l'insertion de ces régions et de ces peuples dans les dynamiques commerciales. Les sociétés d'éleveurs ou d'agro-éleveurs sont relativement traditionnelles, les changements sociaux et les processus de développement à l'échelle locale semblent souvent timides. Néanmoins, la monétarisation de leurs économies, leur réactivité aux signaux du marché et en particulier aux prix sont des processus de mieux en mieux connus (Scoones, 1995; Duteurtre et al., 2003 ; Ancey et al., 2005 ; Vatin, 1996 et 2008).

3 L'internationalisation des produits animaux en Afrique, doublée d'une urbanisation rapide et donc de l'accroissement de la demande locale, offre de nouvelles opportunités marchandes aux éleveurs. Les politiques et les projets d'appui à l'élevage tentent d'utiliser ces ouvertures marchandes pour promouvoir l'intégration économique des 
systèmes d'élevage africains. Les effets de cette intégration marchande sur les dynamiques d'élevage et le développement des régions d'élevage en Afrique est mal connu. Ainsi les accords commerciaux négociés au sein de l'Organisation mondiale du commerce $(\mathrm{OMC})$ et les dynamiques d'intégration régionale ont-ils constitué les principaux arguments justifiant les politiques de réduction des barrières douanières (Hugon, 2002 ; Fao, 2005). L'impact de cette libéralisation a été particulièrement visible dans le secteur de l'élevage. Par exemple, les tarifs à l'importation des produits laitiers, qui avaient été parmi les plus élevés du monde pendant la période de l'Uruguay Round (1986-94), ont été fortement réduits dans le cadre de l'OMC pour atteindre aujourd'hui $25 \%$ en moyenne. Cette baisse est-elle une chance pour les élevages africains qui produisent du lait pour la vente? Rien n'est moins sûr. La réduction des barrières douanières a en effet accéléré l'afflux de lait en poudre sur les marchés urbains africains, processus observé dès les années 1970 (Vatin, 1996) mais qui a pris une ampleur telle que les importations de lait en poudre couvraient par exemple plus de 95\% des besoins de Bamako en 2008, comme le montre Samuel Pinaud dans son travail de thèse en cours dans le cadre d'Icare. Ces importations ne stimulent pas la production locale de lait, elles la concurrencent. Cependant, dans ce contexte de mondialisation, si un facteur vient à perturber les productions exportées à l'échelle mondiale - comme par exemple la sécheresse de 2006 en Australie -, les prix de la poudre de lait peuvent monter (FAO, 2007) et les productions locales de lait s'en trouvent stimulées, et le développement des territoires d'élevage avec. On est là au cœur du questionnement du projet Icare, qui s'applique aussi bien aux dynamiques de commerce de viande qu'à celles du lait, notamment dans la Corne de l'Afrique, où les élevages sont en prise directe avec les politiques d'importation de viande des pays de la péninsule arabique (Djama, 1999).

4 Dans ce contexte d'évolution rapide des marchés des produits animaux, tant du fait de la mondialisation et des fluctuations de prix qui lui sont liées que de l'urbanisation, comment les élevages locaux saisissent-ils les opportunités commerciales qui se présentent à eux, quelles est leur réactivité aux signaux du marché, et dans quelle mesure ces mises en marché participent-elles du développement des territoires d'élevage?

Dans l'espace de cette problématique interdisciplinaire, les géographes étaient en particulier interpellés pour saisir, aux échelles locale et régionale, les dynamiques entre la mise en marché des produits d'élevage et le développement territorial, les économistes et les sociologues travaillant davantage sur les relations entre politiques publiques, incitations commerciales et développement économique d'une production donnée, avec en point central les questions d'accès des éleveurs au marché et de construction sociale de cet accès au marché.

6 Tout d'abord, fallait-il parler de «développement territorial» lié à l'élevage, de développement des territoires d'élevage (mais qu'est-ce qu'un « territoire d'élevage »?) ou plus simplement de développement des territoires où l'élevage est une activité importante (comment, alors, mesurer cette importance ?)? Ces questions se sont posées d'emblée aux géographes impliqués dans le projet Icare, aux chercheurs confirmés comme aux étudiants (Tonneau, 2007).

7 Dans les travaux présentés ci-après, Néné Dia de l'Université Gaston Berger de Saint Louis du Sénégal a eu l'ambition d'attaquer de front ces questions en cherchant à définir le développement territorial sur des espaces de collecte de lait autour de petites 
villes du Sénégal Oriental (Kolda, Vélingara). Ces espaces sont-ils des «territoires du lait » en construction? Et en quoi sont-ils le support de développement territorial, mesuré grâce à des indicateurs qu'elle nous propose?

8 Si on a bien la constitution d'une entité productive ancrée dans l'espace géographique, plus ou moins en réaction à des processus de globalisation et à la montée de concurrences à l'échelle mondiale, en revanche on peut s'interroger dans le cas de ces espaces d'élevage encore largement extensif sur le principe de spécification des actifs (Pecqueur, 2005) ou de révélation de la ressource cachée (Hirschman, 1986, cité par Pecqueur 2005) qui seraient constitutives d'une dynamique territoriale de développement. Quel processus de spécification, consistant selon Pecqueur à «la recherche de la ressource propre au territoire lui permettant de se différencier de son voisin plutôt que d'être en concurrence sur des productions standards ", serait à la base d'un développement territorialisée ? Cela pose in fine la question de la participation des éleveurs à la construction de territoires, défini à la fois comme cadre de régulation, processeur de changement, espaces d'organisation de la production, lieu d'ajustement entre demandes sociales et productions, voire comme outil d'intervention de l'acteur public (Pivot et al, 2003).

Cela pose également la question du lien entre la production des biens et services par les acteurs du territoire, et le débouché marchand pour ces produits qui ne peuvent pas être dissociés dans la construction de ce territoire. La production de lait participe de cette construction dans la périphérie de Kolda. Rentre-t-elle en synergie avec d'autres activités localisées dans le cadre d'une émergence productive et coordonnée dans le cadre d'une construction territoriale? Le papier de Néné Dia nourrit le débat sur la contribution du lait à cette construction.

Les deux autres textes présentés ci-après complètent ce questionnement sous un angle différent, pour ainsi dire transversal. Tandis que Néné Dia part d'espaces d'activité de production, de collecte et de consommation et s'interroge sur la contribution de ces activités à la construction territoriale, Sophie Molina et Géraldine Pinauldt partent de filières d'élevage et analysent leur inscription dans l'espace, sans préjuger de l'existence ou non d'un territoire. Toutes trois analysent les processus de territorialisation de la ressource par des activités d'élevage, et donc en ce sens instruisent des questions de développement territorial. L'entrée retenue par Sophie Molina et Géraldine Pinauldt se situe en amont de ce processus de construction. Elles se sont en effet toutes deux intéressées, dans une perspective historique, aux liens entre les changements dans le contexte sociopolitique de la production de produits animaux et les dynamiques territoriales observables, l'une à l'échelle micro-régional du bassin d'approvisionnement en lait d'une ville (Sophie Molina), l'autre à l'échelle sousrégionale du complexe géographique formé par la corne de l'Afrique de l'Est et la péninsule arabique (Géraldine Pinauldt).

11 Sophie Molina s'interroge ainsi sur les constructions territoriales qui ont conduit progressivement à l'éloignement des villes des élevages extensifs, depuis l'indépendance du Mali, tout d'abord par la volonté gouvernementale d'installer des élites dans des concessions rurales modernes, puis en raison de vagues d'urbanisation, organisées dans un premier temps, puis sauvages. Elle montre ainsi, dans le cas de l'axe Bamako-Koulikoro, qu'entre les fermes de prestige des élites urbaines et les élevages extensifs résiduels, des acteurs se sont engagés dans un processus de spécification de leur territoire grâce au lait, mais que la contribution de ce produit au développement à 
l'échelle locale est encore trop fragile, notamment $d u$ fait de la désorganisation $d u$ réseau de collecte, pour que l'on puisse parler de développement territorial.

Les travaux de Géraldine Pinauldt vont dans le même sens, même s'ils s'inscrivent à une autre échelle qui est celle des concurrences entre Etats africains pour l'accès au marché des pays de la péninsule arabique. Cette concurrence a été exacerbée par la mise en place de quarantaines, notamment à Djibouti, pour faire face aux risques de diffusion hors d'Afrique de la fièvre de la vallée du Rift. L'enjeu commercial est de taille puisque ce ne sont pas moins de 3,5 millions de têtes de bétail qui sont vendues chaque année en direction de la péninsule arabique.

Ces deux contextes sociopolitiques en pleine mutation, en Afrique de l'Est et de l'Ouest, offrent à certains éleveurs de nouvelles possibilités de mise en marché des produits de l'élevage. Ces opportunités donnent-elles lieu à des constructions sociales territorialisées, permettant de révéler, d'ordonnancer voire de transformer les ressources ainsi que le suggère Pecqueur? C'est tout l'objet du débat auquel nous convient ces trois papiers.

\section{BIBLIOGRAPHIE}

Ancey V., Monas G., 2005. « Le pastoralisme au Sénégal, entre politique moderne et gestion des risques par les pasteurs », Tiers monde (184), p. 763-783.

Djama M., 1999. « Producteurs pastoraux et commerce international. L'évolution des rapports marchands en pays Nord-Somali », Bourgeot A. (dir.), Horizons nomades en Afrique Sahélienne. Sociétés, développement et démocratie, Paris, Karthala.

Duteurtre G., Faye B., (dir.), 2003. Elevage et pauvreté. Actes de l'atelier-recherche CIRAD, Montpellier, le 11 et 12 septembre 2003.

FAO, 2005. « Le secteur de l'élevage à l'heure de la mondialisation : incidence des marchés en mutation », Rapport présenté à la XIX ${ }^{\mathrm{e}}$ session du Comité de l'Agriculture, Rome, 13-16 avril 2005, $13 \mathrm{p}$.

FAO, 2007. Perspectives de l'alimentation. Analyse des marchés mondiaux. Système mondial d'information et d'alerte rapide sur l'alimentation et l'agriculture (ftp://ftp.fao.org/docrep/fao/ 010/ah864f/ah864fo0.pdf).

Faye B., 2006. « Les pasteurs sont des éleveurs "contemplatifs" ! », Courade G. (dir.). L'Afrique des idées reçue, Paris, Belin, p. 281-287.

Hirschman A.O., 1986. Vers une économie politique élargie, Paris, Editions de minuit.

Hugon P. (Dir), 2002 : Les économies en développement à l'heure de la régionalisation, Paris, Karthala, $335 \mathrm{p}$.

Pecqueur B., 2005. «Le développement territorial : une nouvelle approche des processus de développement pour les économies du Sud ", Antheaume B., Giraut F., (dir.), Le territoire est mort Vive les territoires, Paris, IRD Editions, p. 295-316. 
Pivot J.-M., Caron P., Bonnal P., 2003. « Coordination locales, actions collectives, territoires et multifonctionnalité de l'agriculture : éclairage et perspectives ", Les cahiers de la multifonctionnalité $\mathrm{n}^{\circ} 3$, p. 4-16.

Scoones I., 1995. Living with uncertainty: new directions in pastoral development in Africa. Intermediate TechnologyPublications, Exeter.

Tonneau J.P., 2007. « Pourquoi diable le développement territorial et, plus encore, pourquoi y croire?", Atelier Méthodologique : Élevage, Commerce et Territoires. Internationalisation des Echanges et Développement Territorial dans les Régions d'Elevage en Afrique, Montpellier (France), CIRAD, 3-4 septembre 2007.

Vatin F., 1996. Le lait et la raison marchande. Essais de sociologie économique, Rennes, Presses Universitaires de Rennes, 205 p.

Vatin F., 2008. « Retour à Dahra. Quelques notes sur le voyage et le développement », Revue du Mauss 2008/2, n 32, p. 441-460.

\section{AUTEUR}

\section{DENIS GAUTIER}

Denis Gautier est chercheur au Cirad. 